\title{
Upregulated Annexin A1 promotes cellular invasion in triple-negative breast cancer
}

\author{
MAIKO OKANO $^{1}$, KENSUKE KUMAMOTO $^{1}$, MOTONOBU SAITO $^{1,2}$, HISASHI ONOZAWA $^{1}$, \\ KATSUHARU SAITO ${ }^{1}$, NORIKO ABE ${ }^{1}$, TOHRU OHTAKE ${ }^{1}$ and SEIICHI TAKENOSHITA ${ }^{1}$ \\ ${ }^{1}$ Department of Organ Regulatory Surgery, Fukushima Medical University School of Medicine, Fukushima 960-1295; \\ ${ }^{2}$ Division of Genome Biology, National Cancer Center Research Institute, Tokyo 104-0045, Japan
}

Received October 1, 2014; Accepted December 3, 2014

DOI: $10.3892 /$ or.2015.3720

\begin{abstract}
Annexin A1 (ANXA1) is a calcium-dependent phospholipid-linked protein, involved in anti-inflammatory effects, regulation of cellular differentiation, proliferation and apoptosis. While many studies have investigated the ANXA1 expression in various tumor types, the role of ANXA1 is not fully understood. Therefore, in the present study, we evaluated the ANXA1 expression in 211 breast cancer patients and compared the levels with clinicopathological factors. ANXA1 was positively expressed in $31(14.7 \%)$ of the 211 cases in our cohort, and these positive cases were associated with triple-negative breast cancer $(\mathrm{TNBC})(\mathrm{P}=0.007)$ and venous invasion $(\mathrm{P}=0.028)$. The in vitro cell experiment found that the MDA-MB-231 cell line, which is a TNBC cell line, highly expressed ANXA1. Using this cell line, the functional role of ANXA1 in breast cancer was revealed and the knockdown of ANXA1 by specific siRNA demonstrated a significant reduction in cellular invasion. Further experiments indicated that ANXA1 was induced by hypoxia with hypoxia-inducible factor-1 $\alpha$ induction. These results suggested that ANXA1, which enhanced breast cancer invasion and metastasis under hypoxia, were significantly associated with the worst patient outcome. This is particularly noted in TNBC, the group of breast cancer with the worst outcome for which new therapeutic implications are required.
\end{abstract}

\section{Introduction}

Breast cancer is a leading cause of cancer related mortality among females worldwide and its incidence and mortality rate have been increasing throughout the recent years (1). The

Correspondence to: Dr Kensuke Kumamoto, Department of Organ Regulatory Surgery, Fukushima Medical University School of Medicine, 1 Hikarigaoka, Fukushima 960-1295, Japan

E-mail:kumamotk@fmu.ac.jp

Key words: breast cancer, ANXA1, cellular invasion, metastasis treatment of breast cancer has progressed over the past three decades with the development of the combination of chemotherapy, endocrine therapies and human epidermal growth factor receptor 2 (HER2)-targeted therapies (2-5). However, triple-negative breast cancer (TNBC), which is defined by the lack of the estrogen receptor (ER), the progesterone receptor $(\mathrm{PgR})$ and HER 2 expression, has not fully benefited from such advances in treatment. Therefore, patients with TNBCs are currently categorized as a sub group with the worst possible outcome. Although recent progress in gene sequencing technology has revealed the genetic profile of breast cancer including that of TNBC, the effort to understand breast cancer based on the diverse view from a more inclusive perspective is still needed (6-8).

Annexin A1 (ANXA1) is a 37-kDa calcium-dependent phospholipid-linked protein belonging to the annexin superfamily and it is related to anti-inflammatory effects, regulation of cellular differentiation, proliferation and apoptosis (9-11). However, through those functions, ANXA1 is involved in tumorigenesis and the pivotal role of ANXA1 is not clearly understood. One of the main reasons for this is due to the fact that the functional role of ANXA1 in malignant tumors is quite different depending on the cancer type, such as head-and-neck, esophageal, gastric, colorectal, pancreatic, hepatic and prostate cancer (12-19). While ANXA1 is highly expressed in malignant tumors $(20,21)$, some studies have demonstrated that lower expression of ANXA1 is associated with poor prognosis (22) and cancer progression (23-25) in breast cancer.

Our recent studies, as well as others, have highlighted the functional role of ANAX1 in cancer progression. One study suggested that ANXA1 may increase metastatic potential through the regulation of NF- $\mathrm{KB}$ in breast cancer (26). Another study reported that ANXA1 may promote metastasis formation by enhancing the TGF $\beta /$ Smad signaling pathway (27). On the other hand, we previously reported that the expression of ANXA1 is upregulated in gastric and colon cancer and it is involved in cancer invasion as well as lymph node metastasis (16). Here, we investigated the ANXA1 expression in breast cancer and the relationship between ANXA1 expression and clinicopathological features. Furthermore, the biological significance of the ANXA1 expression was examined to clarify the function of ANXA1 in breast cancer. 


\section{Materials and methods}

Clinical samples of the patients. Surgically resected 211 breast cancer tissues were examined in the present study. All cases underwent surgical resection at Fukushima Medical University between 2002 and 2005. Information regarding age, TNM stage (the 7th classification), ER, PgR and HER2, and pathological diagnosis including lymphatic and venous invasion, were retrospectively collected. The patient median age was 55 years. These cases included 85 stage I cases, 120 stage II cases and 6 stage III cases. The present study was approved by the Ethics Committee of Fukushima Medical University. Written informed consent was obtained from all participants.

Immunohistochemical (IHC) staining and evaluation. Formalin-fixed paraffin-embedded (FFPE) sections were immunostained for ANXA1, ER, PgR and HER2, and evaluated for staining intensity. Briefly, breast cancer specimens were fixed in a $10 \%$ formalin and embedded in paraffin. They were cut into thin $(4 \mu \mathrm{m})$ sections and stained with hematoxylin and eosin or other antibodies. Analysis of ER, PgR and HER2 was performed by the Ventana HX automatic system BenchMark (Ventana Medical Systems SA, Illkirch Cedex, France). Antibodies used for IHC staining were as follows: anti-ANXA1 (clone 29; BD Biosciences, San Jose, CA, USA), anti-ER (SP1), anti-PgR (1E2), anti-HER2 (4B5) (all from Ventana Medical Systems SA). The expression of ANXA1 protein was evaluated by the ratio of the numbers of positively stained cells to those negatively stained. Tissues were dichotomized as positive ( $\geq 5 \%$ staining) or negative ( $<5 \%$ staining) for ANXA1. ER, PgR and HER2 expression levels were also dichotomized as positive or negative as previously described (28).

Cell line culture. The breast cancer cell lines were originally obtained from the American Type Culture Collection (Rockville, MD, USA) and were cultured in the recommended media with $10 \%$ fetal bovine serum. These monolayer cells were maintained in a $37^{\circ} \mathrm{C}$ incubator with $5 \% \mathrm{CO}_{2}$. Cells were checked regularly under a light microscope and were subcultured when reaching 80 to $90 \%$ confluence. For hypoxia exposure, each cell type was cultured for $24 \mathrm{~h}$ in a modulator incubator chamber (Billups-Rothenberg, Del Mar, CA, USA) at $37^{\circ} \mathrm{C}$ with $1 \% \mathrm{O}_{2}, 5 \% \mathrm{CO}_{2}$ and $94 \% \mathrm{~N}_{2}$. To mimic hypoxia, the cells were cultured for $24 \mathrm{~h}$ with $100 \mu \mathrm{M}$ cobalt chloride $\left(\mathrm{CoCl}_{2}\right)$ (Sigma-Aldrich, St. Louis, MO, USA).

Western blotting. Cells were washed twice with ice-cold PBS and immediately scraped. The cells were then centrifuged at 15,000 rpm to pellet cellular debris and were stored overnight at $-80^{\circ} \mathrm{C}$. The protein lysates were collected using ice-cold RIPA buffer containing Halt Protease Inhibitor Single-Use Cocktail (Thermo Scientific, San Jose, CA, USA). The protein concentration was determined by the Bradford method (Bio Rad, Hercules, CA, USA). Samples for SDS-PAGE were prepared by mixing aliquots of the protein with Novex Tris-Glycine SDS sample buffer (Invitrogen, Carlsbad, CA, USA) and heated at $100^{\circ} \mathrm{C}$ for $3 \mathrm{~min}$. Protein samples were run on $10 \%$ Bis-Tris gels at $100 \mathrm{~V}$ for $90 \mathrm{~min}$ with MES SDS running buffer (Invitrogen). For western blotting, the gels were electro-transferred to a nitrocellulose membrane using the iBlot Dry Blotting System (Invitrogen). The proteins were blocked using Starting Block (PBS) Blocking Buffer (Pierce, Rockford, IL, USA) and were detected using anti-ANXA1, anti-hypoxia-inducible factor-1 $\alpha$ (HIF$1 \alpha)$ (BD Novus Bioscience Biologicals, Littleton, CO, USA), anti- $\beta$-actin (Santa Cruz Biotechnology, Dallas, TX, USA) and a goat anti-mouse secondary antibody phosphatase (Novagen, Billerica, MA, USA). Western blottings were then incubated with Super Signal West Pico detection system (Pierce) and detected using LAS-4000 IR MultiColor (Fuji, Tokyo, Japan).

Quantitative reverse transcription-polymerase chain reaction ( $q R T-P C R)$. The total RNA was extracted from the cells using TRIzol reagent (Invitrogen) according to the manufacturer's instructions. Complementary DNA (cDNA) was synthesized from $5 \mu \mathrm{g}$ of total RNA with a random hexamer using the SuperScript III First-Strand Synthesis kit (Invitrogen). These cDNAs were used for the measurement of gene expression with a 7500 Real-time PCR system (Applied Biosystems, Foster City, CA, USA) using TaqMan probes, and experiments were performed in triplicate to the blinded patient information. $\beta$-actin was used as an internal control. Expression assays were purchased from Applied Biosystems: ANXA1 (Hs00167549_m1) and $\beta$-actin (Hs99999903_m1). Normalized ANXA1 gene expression was calculated using the $2^{-\Delta \Delta C \mathrm{CT}}$ method as previously described (29).

Knockdown of ANXAl expression using siRNA technology. The siRNA oligonucleotides for ANXA1 (HSS100502 and HSS100503) and the control were purchased from Invitrogen. ANXA1 siRNA sequences were designed as follows: siRNA-1, 5'-CAACCAUCAUUGACAUUCUAACUAA-3' and siRNA-2, 5'-GCCUUGCAUAAGGCCAUAAUGGUUA-3'. One day prior to the siRNA transfection, $5 \times 10^{5}$ cells were plated in $6 \mathrm{~cm}$ plates. The cells were transfected with $40 \mathrm{nM}$ of each siRNA using Lipofectamine RNAiMAX (Invitrogen) following the manufacturer's instructions and harvested after $48 \mathrm{~h}$.

Invasion assay. The cell invasion assay was conducted using a 24-well BD Bio-Coat Tumor Invasion System (BD Biosciences). Cells were harvested and seeded $\left(5 \times 10^{3}\right.$ cells $)$ into the top chamber and incubated at $37^{\circ} \mathrm{C}$ for $22 \mathrm{~h}$. The cells were post-stained with $4 \mu \mathrm{g} / \mathrm{ml}$ of Calcein-AM (Molecular Probes, Carlsbad, CA, USA) in Hank's buffered salt solution at $37^{\circ} \mathrm{C}$ in $5 \% \mathrm{CO}_{2}$ for $1 \mathrm{~h}$. The labeled cells that invaded the BD Matrigel Matrix and passed through the pores of the BD FluoroBlok membrane were detected by Varioskan Flash Multimode Reader (Thermo Scientific).

Statistical data analysis. Statistical analysis was carried out with an unpaired Student's t-test using GraphPad Prism v5.0 (GraphPad Software, Inc., La Jolla, CA, USA). P $<0.05$ was considered to indicate a statistically significant result.

\section{Results}

ANXA1 protein expression in breast cancer tissues. The ANXA1 protein expression in the breast cancer tissues was 

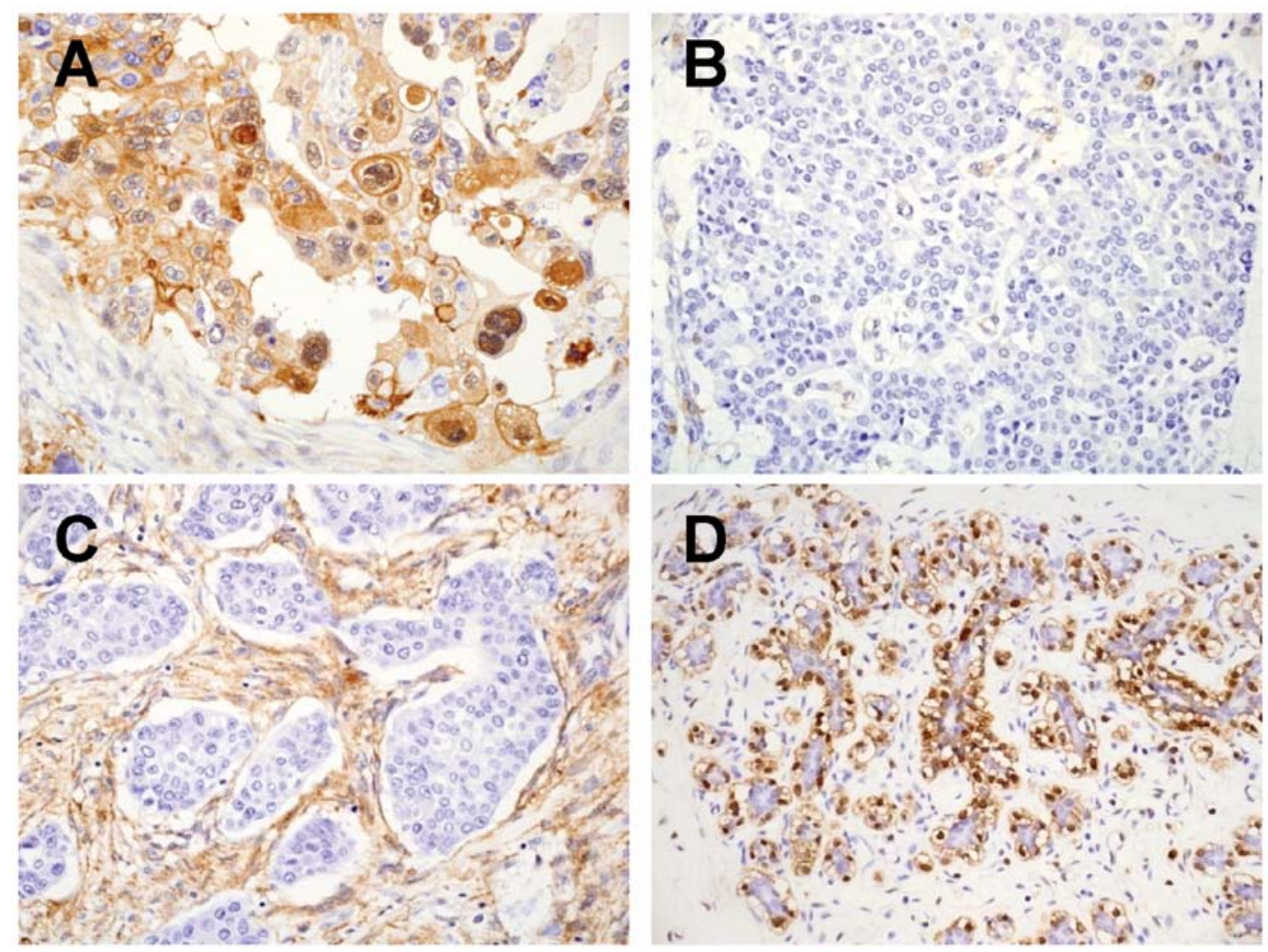

Figure 1. Representative images of ANXA1 immunohistochemical staining in breast cancer. (A and B) Positive and negative ANXA1 staining in breast cancer tissue. (C) Positive ANXA1 staining of interstitial cells in breast cancer tissue. (D) Positive ANXA1 staining of myoepithelial cells in adjacent normal breast tissue. x400 magnification. ANXA1, Annexin A1.

examined by IHC staining using an anti-ANXA1 antibody. In the breast cancer tissues, the ANXA1 expression was mainly detected in the cytoplasm of tumor cells, interstitial cells and myoepithelial cells (Fig. 1). In contrast, in the adjacent normal breast tissues, the ANXA1 expression was detected in myoepithelial cells, but not in normal duct-epithelial cells.

Of the 211 cases, 31 (14.7\%) showed positive ANXA1 staining in the breast cancer tissues. Such cases showed a positive correlation with triple-negative (ER-negative, PgR-negative and HER2-negative) expression ( $\mathrm{P}=0.007)$ and venous invasion $(\mathrm{P}=0.028)$ (Table I). However, such cases did not show any correlation with age, TNM stage, lymph node metastasis, histology, ER and PgR expression and recurrence. These results suggest that positive expression of ANXA1 is associated with tumor cell invasion in breast cancer, which was particularly significant in the TNBC cases.

ANXA1 expression in the breast cancer cell lines. We next examined the ANXA1 expression in five human breast cancer cell lines, MCF-7, T-47D, SK-Br-3, ZR-75-1 and MDA-MB-231. IHC staining using the anti-ANXA1 antibody was performed for those cell lines. Positive staining of ANXA1 was detected in the cytoplasm of the MDA-MB-231 cells, while that of ANXA1 was not detected in the other four cell lines (Fig. 2A). We also performed IHC staining and evaluation for ER, PgR and HER2 in the same breast cancer cell lines. Positive staining of both ER and PgR was observed in the MCF-7 and T-47D cells. Strong positive staining of HER2 was detected in the SK-Br-3 cells and weak positive staining of HER2 was detected in the ZR-75-1 cells. As a result, the MDA-MB-231 cell line was found to be a TNBC with ANXA1-positive expression.

To confirm the ANXA1 expression by IHC evaluation in the cancer cell lines, we performed western blotting and qRT-PCR using the same breast cancer cell lines. Consistent with the IHC results, the ANXA1 protein was highly expressed in the MDA-MB 231 cells (Fig. 2B). The mRNA of ANXA1 was also highly expressed in MDA-MB-231 cells, suggesting that the ANXA1 expression was upregulated at the transcriptional level (Fig. 2C).

Knockdown of ANXA1 in the MDA-MB-231 cells. According to the comparisons of our IHC results and the clinicopathological factors, we hypothesized that ANXA1 is upregulated in TNBCs and is associated with cellular invasion. To investigate this hypothesis, we used gene knockdown technology and cell invasion assay. Both protein and mRNA expression of ANXA1 was downregulated by two independent siRNA oligonucleotides (siRNA-1 and -2) in the MDA-MB-231 cells which originally exhibited upregulated ANXA1 (Fig. 3A and B). While no morphological changes were observed in the ANXA1 knockdown cells, the ability of cellular invasion was attenuated in the ANXA1 downregulated cells. The invasion ratio was significantly decreased in the cells treated by both ANXA1 siRNA-1 $(\mathrm{P}<0.05)$ and siRNA-2 $(\mathrm{P}<0.01)$ (Fig. 3C).

Induction of ANXA1 in hypoxia. To further assess the induction of ANXA1 during cancer progression, we evaluated ANXA1 
Table I. Clinicopathological factors and ANXA1 expression in 211 breast cancer cases.

\begin{tabular}{|c|c|c|c|c|}
\hline \multirow[b]{2}{*}{ Characteristics } & \multirow[b]{2}{*}{ Total $(n=211)$} & \multicolumn{2}{|c|}{ ANXA1 } & \multirow[b]{2}{*}{ P-value } \\
\hline & & $\begin{array}{c}\text { Positive }(\mathrm{n}=31) \\
\mathrm{n}(\%)\end{array}$ & $\begin{array}{c}\text { Negative }(\mathrm{n}=180) \\
\mathrm{n}(\%)\end{array}$ & \\
\hline Age (years) & & & & 1 \\
\hline$<50$ & 75 & $11(35)$ & $64(36)$ & \\
\hline$\geq 50$ & 136 & $20(65)$ & $116(64)$ & \\
\hline pTMN stage & & & & 0.597 \\
\hline $\mathrm{I}$ & 85 & $11(35)$ & $74(41)$ & \\
\hline II & 120 & $20(65)$ & $100(56)$ & \\
\hline III & 6 & 0 & $6(3)$ & \\
\hline Lymph node metastasis & & & & 0.318 \\
\hline Positive & 82 & $15(48)$ & $67(37)$ & \\
\hline Negative & 129 & $16(52)$ & $113(63)$ & \\
\hline Histology & & & & 1 \\
\hline Invasive ductal & 186 & $28(90)$ & $158(88)$ & \\
\hline Invasive lobular & 7 & $1(3)$ & $6(3)$ & \\
\hline Mixed & 2 & 0 & $2(1)$ & \\
\hline Other & 16 & $2(7)$ & $14(8)$ & \\
\hline Histological grade & & & & 0.055 \\
\hline $0-2$ & 181 & $23(74)$ & $158(88)$ & \\
\hline 3 & 30 & $8(26)$ & $22(12)$ & \\
\hline Estrogen receptor & & & & 0.09 \\
\hline Positive & 150 & $18(58)$ & $132(73)$ & \\
\hline Negative & 61 & $13(42)$ & $48(27)$ & \\
\hline Progesterone receptor & & & & 0.432 \\
\hline Positive & 124 & $16(52)$ & $108(60)$ & \\
\hline Negative & 87 & $15(48)$ & $72(40)$ & \\
\hline HER2 & & & & 1 \\
\hline Positive & 16 & $2(7)$ & $14(8)$ & \\
\hline Negative & 195 & $29(93)$ & $166(92)$ & \\
\hline Triple-negative & & & & 0.007 \\
\hline Presence & 35 & $11(35)$ & $24(13)$ & \\
\hline Absence & 176 & $20(65)$ & $156(87)$ & \\
\hline Lymphatic invasion & & & & 0.329 \\
\hline Positive & 116 & $20(65)$ & $96(53)$ & \\
\hline Negative & 95 & $11(35)$ & $84(47$ & \\
\hline Venous invasion & & & & 0.028 \\
\hline Positive & 58 & $14(45)$ & $44(24)$ & \\
\hline Negative & 153 & $17(55)$ & $136(76)$ & \\
\hline Recurrence & & & & 0.088 \\
\hline Presence & 20 & $6(19)$ & $14(8)$ & \\
\hline Absence & 191 & $25(81)$ & $166(92)$ & \\
\hline
\end{tabular}

P-value from Fisher's exact test. ANXA1, Annexin A1. Significant P-value are indicated in bold.

induction under hypoxic conditions, which is one of the main characteristic features of a tumor. T-47D and SK-Br3 breast cancer cell lines were treated with hypoxia and the hypoxia mimic induced by $\mathrm{CoCl}_{2}$. HIF-1 $\alpha$ protein was induced in both cell lines (Fig. 4A). The ANXA1 protein was also induced by hypoxia in both cell lines. This induction of ANXA1 was also 

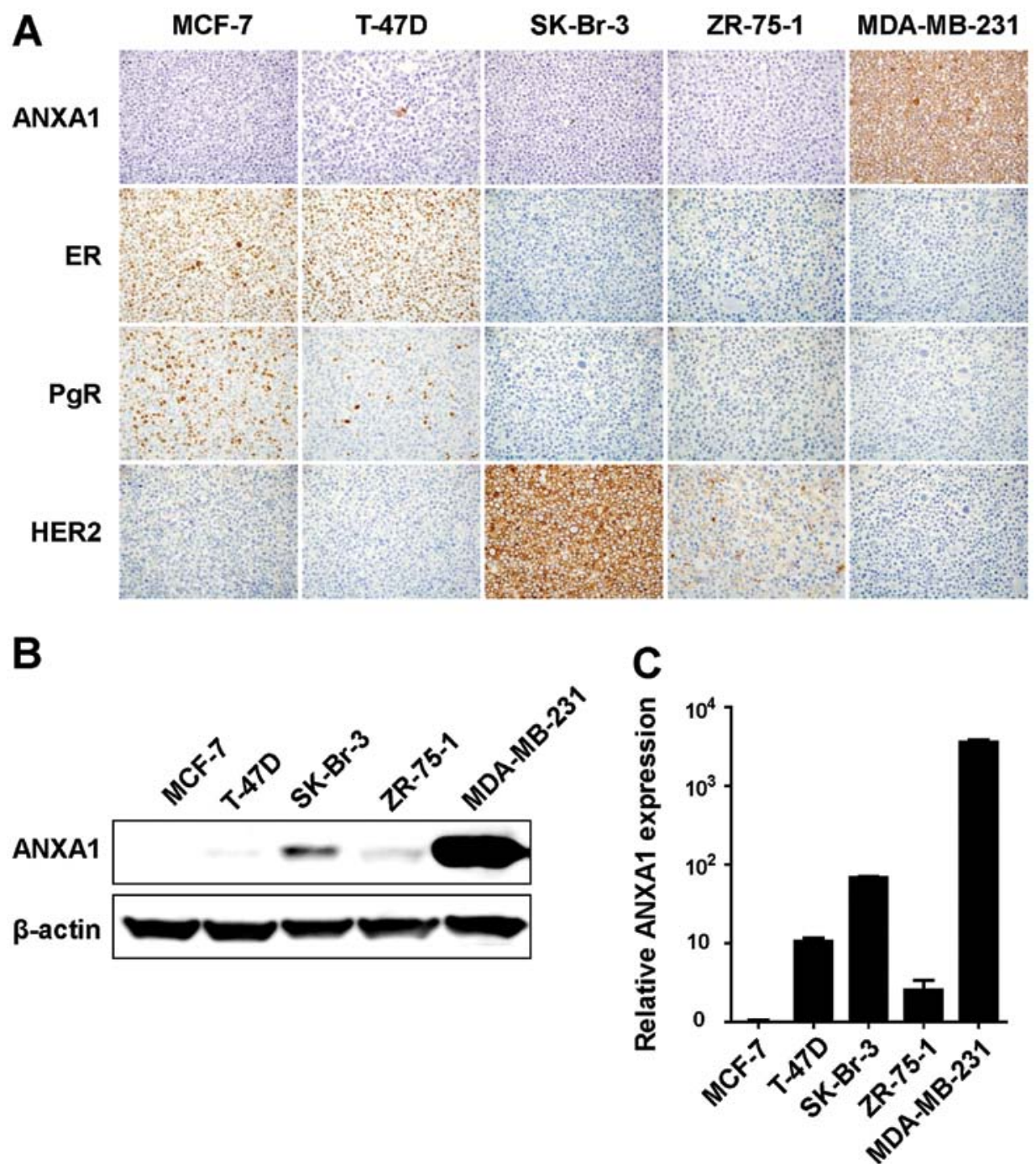

Figure 2. ANXA1 expression in breast cancer cell lines. (A) Immunohistochemical staining of ANXA1, ER, PgR and HER2 in human breast cancer cell lines. Magnification, $x$ 400. (B) Western blotting of the ANXA1 expression in breast cancer cell lines. Cell lysates from each cell line were examined by western blotting using the anti-ANXA1 antibody. $\beta$-actin was used as a loading control. (C) qRT-PCR analysis of the ANXA1 expression in breast cancer cell lines. Relative ANXA1 expression levels to MCF-7 (MCF-7=1) are shown (normalized to $\beta$-actin). Data are mean \pm SD. ANXA1, Annexin A1; HER2, epidermal growth factor receptor; ER, estrogen receptor; PgR, progesterone receptor; qRT-PCR, quantitative reverse transcription polymerase chain reaction.

confirmed by examining mRNA expression (Fig. 4B). These results suggested that hypoxia affects tumor invasion through induction of ANXA1 in TNBC.

\section{Discussion}

Although it has been reported that ANXA1 plays an important role in tumor development and progression, due to the fact that the expression pattern of ANXA1 is different across tumor types, the exact mechanisms of ANXA1 in cancer remain unknown. While the ANXA1 expression on cancer tissues is downregulated in head and neck, esophageal and prostate cancer, the expression is upregulated in stomach, colorectal, pancreas and hepatic cancer (12-19). In breast cancer, the expression and functional roles of ANXA1 are controversial. Some reports describe that cancer cells show positive ANXA1 expression, whereas nonmalignant cells do not express ANXA1, except for myoepithelial cells in normal tissues $(20,21)$. In vivo experiments using rats showed that ANXA1 was highly expressed in lung metastatic tumors compared to parental cells (20). However, other studies demonstrated that low expression of ANXA1 was associated with poor prognosis (22) and cancer progression (23-25).

In the present study, we evaluated the ANXA1 expression in breast cancer tissues and detected positive expression of ANXA1 in 31 (14.7\%) of the 211 cases. Although the positive ANXA1 cases appeared to have a relatively smaller ratio, those cases had a significant correlation with TNBC and venous invasion, suggesting that ANXA1 is associated with poor patient outcome and malignant potential. We also evaluated the ANXA1 expression in several breast cancer cell lines. In the five breast cancer cell lines, MDA-MB-231 cells highly expressed ANXA1 without ER, PgR or HER2 expression. These cells are well known for their invasive and metastatic potential (27,30-32). Therefore, we investigated the biological significance of ANXA1 by using knockdown methods in the MDA-MB-231 cells. As shown in Fig. 3C, suppression of the ANXA1 expression resulted in a significant reduction of the cellular invasion ability, showing the strong malignant potential of ANXA1. 
A

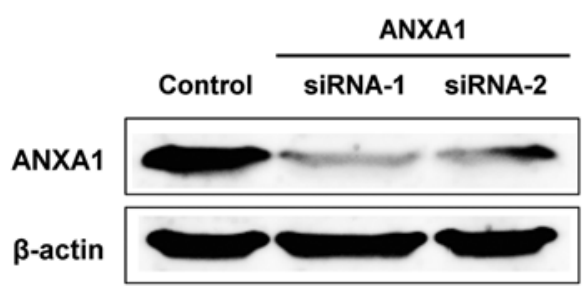

B

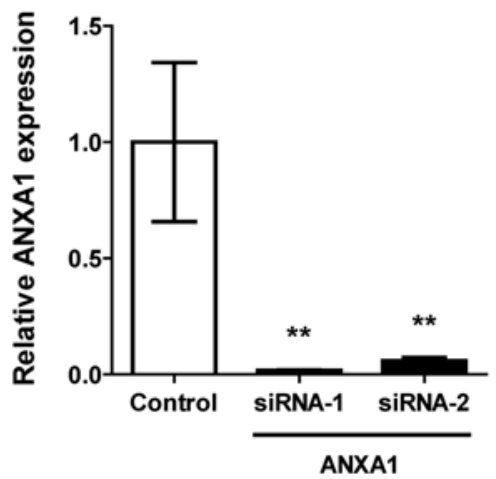

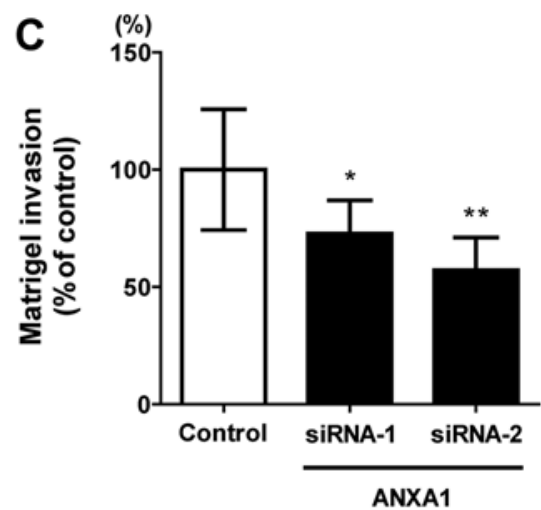

Figure 3. Knockdown of ANXA1 in MDA-MB-231 cells. Control siRNA and two independent siRNAs (siRNA-1 and -2) were used. (A) siRNA-transfected MDA-MB-231 cell lysates were examined by western blotting using the anti-ANXA1 antibody. $\beta$-actin was used as a loading control. (B) qRT-PCR analysis of ANXA1 expression in siRNA-transfected MDA-MB-231 cells. Relative ANXA1 expression levels are shown (normalized to $\beta$-actin). Data are mean \pm SD ${ }^{* *} \mathrm{P}<0.01$. (C) A cell invasion assay was performed using a 24 -well BD BioCoat Tumor Invasion System. Data are mean \pm SD. ${ }^{*} \mathrm{P}<0.05$ and ${ }^{* *} \mathrm{P}<0.01$. ANXA1, Annexin A1.
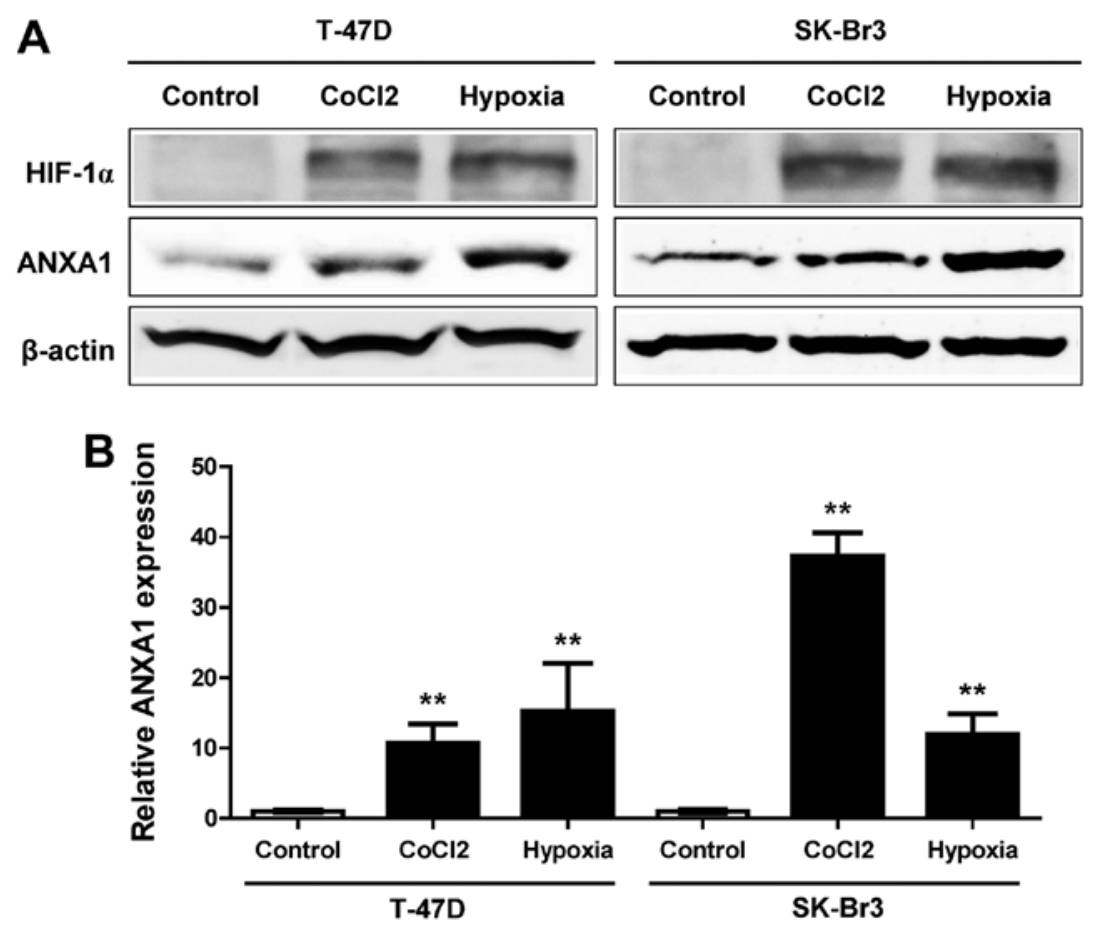

Figure 4. Hypoxia treatment in the T-47D and SK-Br-3 cells. (A) Cell as lysates with hypoxia and hypoxia mimic induced by $\mathrm{CoCl}_{2}$ were examined by western blotting using the anti-HIF-1 $\alpha$ and anti-ANXA1 antibodies. $\beta$-actin was used as a loading control. (B) qRT-PCR analysis of ANXA1 expression in both cells with hypoxia. Relative ANXA1 expression levels to the control (control=1) are shown (normalized to $\beta$-actin). Data are mean $\pm \mathrm{SD}$. ${ }^{* *} \mathrm{P}<0.01$. ANXA1, Annexin A1.

In addition, MDA-MB-231 cells show a multidrug resistance due to upregulated ANXA1 expression $(33,34)$. This cell line shows significant resistance to adriamycin, melphalan and etoposide compared to MCF-7 cells, which lack ANXA1 expression. Drug resistance is also related to hypoxia of malignant tumors (35). Hypoxia inhibits tumor cell proliferation, induces cell cycle arrest and affects the expression of various proteins, resulting in the reduction of chemotherapeutic effects (36). For example, HIF-1 $\alpha$, a transcription factor that contains a basic helix-loop-helix motif as well as a PAS domain, is increased by hypoxia in various types of cancers and precancerous lesions (37-40). HIF-1 $\alpha$ promotes tumor progression through direct or indirect interactions with important oncogenes and tumor suppressor genes, such as MYC and TP53 (41). Hypoxia and the hypoxia mimic induced by $\mathrm{CoCl}_{2}$ also increased ANXA1 expression in both the T-47D and SK-Br-3 breast cancer cell lines. As HIF inhibitors are currently undergoing clinical evaluation as a useful treatment for cancer, simultaneous inhibition of ANXA1 with HIF inhibitors may increase the antitumor effects in breast cancer. Taken together, our results indicate that the clinical significance of ANXA1 expression may be related to the tumor characteristics of TNBC, which is of high importance for developing new treatments. 
Our large-scale examination could make a major contribution to the understanding of the role of ANXA1. Although further investigation is necessary, our results are a first step into the new ANXA1 aspect in breast cancer.

\section{References}

1. Jemal A, Bray F, Center MM, Ferlay J, Ward E and Forman D: Global cancer statistics. CA Cancer J Clin 61: 69-90, 2011.

2. Early Breast Cancer Trialists' Collaborative Group, Davies C, Godwin J, et al: Relevance of breast cancer hormone receptors and other factors to the efficacy of adjuvant tamoxifen: patient-level meta-analysis of randomised trials. Lancet 378: 771-784, 2011.

3. Early Breast Cancer Trialists' Collaborative Group, Peto R, Davies C, et al: Comparisons between different polychemotherapy regimens for early breast cancer: meta-analyses of long-term outcome among 100,000 women in 123 randomised trials. Lancet 379: 432-444, 2012.

4. Gianni L, Dafni U, Gelber RD, et al: Treatment with trastuzumab for 1 year after adjuvant chemotherapy in patients with HER2-positive early breast cancer: a 4-year follow-up of a randomised controlled trial. Lancet Oncol 12: 236-244, 2011.

5. Cortazar P, Zhang L, Untch M, et al: Pathological complete response and long-term clinical benefit in breast cancer: the CTNeoBC pooled analysis. Lancet 384: 164-172, 2014.

6. Cancer Genome Atlas Network: Comprehensive molecular portraits of human breast tumours. Nature 490: 61-70, 2012.

7. Turner NC and Reis-Filho JS: Tackling the diversity of triple-negative breast cancer. Clin Cancer Res 19: 6380-6388, 2013.

8. Cancer Genome Atlas Research Network; Kandoth C, Schultz N, et al: Integrated genomic characterization of endometrial carcinoma. Nature 497: 67-73, 2013.

9. Rescher U and Gerke V: Annexins - unique membrane binding proteins with diverse functions. J Cell Sci 117: 2631-2639, 2004.

10. Parente L and Solito E: Annexin 1: more than an anti-phospholipase protein. Inflamm Res 53: 125-132, 2004.

11. Lim LH and Pervaiz S: Annexin 1: the new face of an old molecule. FASEB J 21: 968-975, 2007.

12. Garcia Pedrero JM, Fernandez MP, Morgan RO, et al: Annexin A1 down-regulation in head and neck cancer is associated with epithelial differentiation status. Am J Pathol 164: 73-79, 2004.

13. Luo A, Kong J, Hu G, et al: Discovery of $\mathrm{Ca}^{2}{ }^{+}$-relevant and differentiation-associated genes downregulated in esophageal squamous cell carcinoma using cDNA microarray. Oncogene 23: 1291-1299, 2004

14. Sinha P, Hütter G, Köttgen E, Dietel M, Schadendorf D and Lage H: Increased expression of annexin I and thioredoxin detected by two-dimensional gel electrophoresis of drug resistant human stomach cancer cells. J Biochem Biophys Methods 37: 105-116, 1998

15. Duncan R, Carpenter B, Main LC, Telfer C and Murray GI: Characterisation and protein expression profiling of annexins in colorectal cancer. Br J Cancer 98: 426-433, 2008.

16. Sato Y, Kumamoto K, Saito K, et al: Up-regulated Annexin A1 expression in gastrointestinal cancer is associated with cancer invasion and lymph node metastasis. Exp Ther Med 2: 239-243, 2011.

17. Bai XF, Ni XG, Zhao P, et al: Overexpression of annexin 1 in pancreatic cancer and its clinical significance. World J Gastroenterol 10: 1466-1470, 2004.

18. Masaki T, Tokuda M, Ohnishi M, et al: Enhanced expression of the protein kinase substrate annexin in human hepatocellular carcinoma. Hepatology 24: 72-81, 1996.

19. Kang JS, Calvo BF, Maygarden SJ, Caskey LS, Mohler JL and Ornstein DK: Dysregulation of annexin I protein expression in high-grade prostatic intraepithelial neoplasia and prostate cancer. Clin Cancer Res 8: 117-123, 2002.

20. Pencil SD and Toth M: Elevated levels of annexin I protein in vitro and in vivo in rat and human mammary adenocarcinoma. Clin Exp Metastasis 16: 113-121, 1998.

21. Ahn SH, Sawada H, Ro JY and Nicolson GL: Differential expression of annexin I in human mammary ductal epithelial cells in normal and benign and malignant breast tissues. Clin Exp Metastasis 15: 151-156, 1997.
22. Wang LP, Bi J, Yao C, et al: Annexin A1 expression and its prognostic significance in human breast cancer. Neoplasma 57: 253-259, 2010.

23. Shen D, Chang HR, Chen Z, et al: Loss of annexin A1 expression in human breast cancer detected by multiple highthroughput analyses. Biochem Biophys Res Commun 326: 218-227, 2005

24. Shen D, Nooraie F, Elshimali Y, et al: Decreased expression of annexin A1 is correlated with breast cancer development and progression as determined by a tissue microarray analysis. Hum Pathol 37: 1583-1591, 2006.

25. Cao Y, Li Y, Edelweiss M, et al: Loss of annexin A1 expression in breast cancer progression. Appl Immunohistochem Mol Morphol 16: 530-534, 2008

26. Bist P, Leow SC, Phua QH, et al: Annexin-1 interacts with NEMO and RIP1 to constitutively activate IKK complex and NF- $\kappa$ B: implication in breast cancer metastasis. Oncogene 30: 3174-3185, 2011.

27. de Graauw M, van Miltenburg MH, Schmidt MK, et al: Annexin A1 regulates TGF-beta signaling and promotes metastasis formation of basal-like breast cancer cells. Proc Natl Acad Sci USA 107: 6340-6345, 2010.

28. Saito M, Matsuzaki M, Sakuma T, et al: Clinicopathological study of non-palpable familial breast cancer detected by screening mammography and diagnosed as DCIS. Breast Cancer 21: 140-145, 2014.

29. Saito M, Shiraishi K, Matsumoto K, et al: A three-microRNA signature predicts responses to platinum-based doublet chemotherapy in patients with lung adenocarcinoma. Clin Cancer Res 20: 4784-4793, 2014.

30. Mastro AM and Vogler EA: A three-dimensional osteogenic tissue model for the study of metastatic tumor cell interactions with bone. Cancer Res 69: 4097-4100, 2009.

31. Nicolson GL, Nawa A, Toh Y, Taniguchi S, Nishimori K and Moustafa A: Tumor metastasis-associated human MTA1 gene and its MTA1 protein product: role in epithelial cancer cell invasion, proliferation and nuclear regulation. Clin Exp Metastasis 20: 19-24, 2003.

32. Chen WT, Lee CC, Goldstein L, et al: Membrane proteases as potential diagnostic and therapeutic targets for breast malignancy. Breast Cancer Res Treat 31: 217-226, 1994.

33. Wang Y, Serfass L, Roy MO, Wong J, Bonneau AM and Georges E: Annexin-I expression modulates drug resistance in tumor cells. Biochem Biophys Res Commun 314: 565-570, 2004.

34. Chuthapisith S, Bean BE, Cowley G, et al: Annexins in human breast cancer: Possible predictors of pathological response to neoadjuvant chemotherapy. Eur J Cancer 45: 1274-1281, 2009.

35. Gottesman MM: Mechanisms of cancer drug resistance. Annu Rev Med 53: 615-627, 2002.

36. Gardner LB, Li Q, Park MS, Flanagan WM, Semenza GL and Dang CV: Hypoxia inhibits G1/S transition through regulation of p27 expression. J Biol Chem 276: 7919-7926, 2001.

37. Giatromanolaki A, Koukourakis MI, Sivridis E, et al: Relation of hypoxia inducible factor 1 alpha and 2 alpha in operable non-small cell lung cancer to angiogenic/molecular profile of tumours and survival. Br J Cancer 85: 881-890, 2001.

38. Liu L, Ning X, Sun L, et al: Hypoxia-inducible factor-1 alpha contributes to hypoxia-induced chemoresistance in gastric cancer. Cancer Sci 99: 121-128, 2008.

39. Schindl M, Schoppmann SF, Samonigg H, et al: Overexpression of hypoxia-inducible factor lalpha is associated with an unfavorable prognosis in lymph node-positive breast cancer. Clin Cancer Res 8: 1831-1837, 2002.

40. Welsh SJ, Bellamy WT, Briehl MM and Powis G: The redox protein thioredoxin-1 (Trx-1) increases hypoxia-inducible factor 1alpha protein expression: Trx-1 overexpression results in increased vascular endothelial growth factor production and enhanced tumor angiogenesis. Cancer Res 62: 5089-5095, 2002.

41. Keith B, Johnson RS and Simon MC: HIF1 $\alpha$ and HIF2 $\alpha$ : sibling rivalry in hypoxic tumour growth and progression. Nat Rev Cancer 12: 9-22, 2012. 